Journal of Nepal Mathematical Society (JNMS), Vol. 2, Issue 2 (2019); Ching-I Hsin

\title{
Inner Finite Order Automorphisms of Type $A$
}

\author{
Ching-I Hsin \\ Department of Multimedia and Game Development, Minghsin University of Science and Technology, Hsinchu County, \\ Taiwan \\ Email: chingihsin@gmail.com
}

\begin{abstract}
Let $\mathfrak{g}$ be a complex simple Lie algebra, and let aut $(\mathfrak{g})$ be the group of all automorphisms on $\mathfrak{g}$. The finite order members of aut( $\mathfrak{g})$ have been classified by Kac, up to conjugation by $\mathfrak{g}$-automorphisms. In other words, this classification does not distinguish two finite order $\mathfrak{g}$-automorphisms $\sigma, \tau$ if there exists $u \in \operatorname{aut}(\mathfrak{g})$ such that $\sigma=u^{-1} \tau u$. For $\mathfrak{g}=\mathfrak{s l}(n, \mathbb{C})$ and $\sigma, \tau$ inner, we provide a finer classification up to conjugation by inner $\mathfrak{g}$-automorphisms, namely we do not distinguish $\sigma, \tau$ only if $u$ is an inner automorphism.
\end{abstract}

Keywords: complex simple Lie algebras, inner automorphisms.

\section{Introduction}

Let $\mathfrak{g}$ be a complex simple Lie algebra, and let aut $(\mathfrak{g})$ denote the $\mathfrak{g}$-automorphisms. The finite order $\mathfrak{g}$ automorphisms play important roles in algebra, such as the construction of Kac-Moody algebras. For this reason, they are well-studied and classified by Kac [4], which prompts generalization in the super setting by Chuah [1. Kac's classification is up to conjugation by $\mathfrak{g}$-automorphism, namely it does not distinguish $\sigma, \tau \in \operatorname{aut}(\mathfrak{g})$ if there exists $u \in \operatorname{aut}(\mathfrak{g})$ such that $\sigma=u^{-1} \tau u$. Here aut $(\mathfrak{g})$ is a Lie group, and we let int $(\mathfrak{g})$ denote the inner $\mathfrak{g}$-automorphisms, namely the $\mathfrak{g}$-automorphisms in the identity component of aut( $\mathfrak{g})$. One may consider the classification under a stricter notion of conjugation, by requiring $u \in \operatorname{int}(\mathfrak{g})$ in above. Obviously this concerns only $\mathfrak{g}$ of types $A, D$ and $E_{6}$, since they are the only cases where $\operatorname{int}(\mathfrak{g}) \neq \operatorname{aut}(\mathfrak{g})$ (this is equivalent to the existence of nontrivial symmetry on the Dynkin diagram of $\mathfrak{g}$ ). In this article, we classify the finite order inner automorphisms on $\mathfrak{s l}(n, \mathbb{C})=A_{n-1}$ up to conjugation by inner automorphisms.

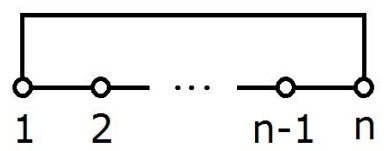

Figure 1: Extended Dynkin diagram of $\mathfrak{s l}(n, \mathbb{C})$.

Let $\mathfrak{g}=\mathfrak{s l}(n, \mathbb{C})$. Its extended Dynkin diagram is as given in Figure 1. Let $D$ denote the diagram in Figure 1 , with vertices labeled $1, \ldots, n$ as indicated. A Kac diagram $c$ is an assignment of nonnegative integers $c_{j}$ to the vertices $j$ of $D$, such that $c_{1}, \ldots, c_{n}$ have no nontrivial common factor. Let $\mathcal{K}$ denote all the Kac diagrams.

Let $c$ be a Kac diagram. Let $m=\sum_{1}^{n} c_{j}$, and let $\omega=\exp (2 \pi i) / m \in \mathbb{C}$. The vertices $\alpha \in D$ represent the union of a simple system and its lowest roots.

Theorem 1.1. (Kac) [2, Ch.X-5,Thm.5.15] [4, Ch.8] A Kac diagram c represents a $\mathfrak{g}$-automorphism $\sigma$ of order $m$, where $\sigma$ acts as multiplication by $\omega^{c_{\alpha}}$ on the root space represented by $\alpha \in D$. Up to conjugation by $\operatorname{aut}(\mathfrak{g})$, all finite order inner $\mathfrak{g}$-automorphisms are obtained in this way.

The general Kac's theorem deals with all finite order automorphisms on complex simple Lie algebras. Here we focus only on finite order inner automorphisms on $\mathfrak{s l}(n, \mathbb{C})$.

In the above theorem, "up to conjugation" means that it does not distinguish $\sigma, \tau$ if they are related by $\sigma=u^{-1} \tau u$ for some $u \in \operatorname{aut}(\mathfrak{g})$. It also does not distinguish two Kac diagrams $c, d \in \mathcal{K}$ if they are related by a symmetry on $D$. In this article, we study a stricter condition, namely we do not distinguish $\tau$ and 
$u^{-1} \tau u$ only if $u$ is an inner automorphism. We shall see in the theorem below that a stricter condition is imposed on $\mathcal{K}$, namely we do not distinguish $c, d \in \mathcal{K}$ only if they are related by the cyclic group $\mathbb{Z}_{n}$ in the symmetry group of $D$.

The symmetry group of $D$ is the dihedral group $\mathbb{D}_{n}=\mathbb{Z}_{n} \rtimes \mathbb{Z}_{2}$, where the normal subgroup $\mathbb{Z}_{n}$ acts as rotations and $\mathbb{Z}_{2}$ contains a nontrivial reflection. It induces a $\mathbb{D}_{n}$-action on $\mathcal{K}$ by

$$
(g c)_{j}=c_{g(j)} ; g \in \mathbb{D}_{n}, c \in \mathcal{K} \text {. }
$$

Theorem 1.2. Two inner finite order $\mathfrak{g}$-automorphisms are conjugate by inner $\mathfrak{g}$-automorphisms if and only if their Kac diagrams are in the same $\mathbb{Z}_{n}$-orbit.

Theorem 1.2 is the main result of this article. In Section 2, we prove Theorem 1.2 In Section 3, we provide some examples to illustrate the ideas.

\section{$2 \quad$ Kac Diagrams of $\mathfrak{s l}(n, \mathbb{C})$}

Recall that $D$ is the extended Dynkin diagram of $\mathfrak{s l}(n, \mathbb{C})$ with vertices labeled as $1, \ldots, n$ as in Figure 1. Let $\mathcal{K}$ be the Kac diagrams on $D$. In Kac's Theorem 1.1, he does not distinguish two Kac diagrams which are related by a symmetry on $D$. However we consider two such diagrams as distinct members of $\mathcal{K}$. So for example Figure 3(b) and Figure $3(\mathrm{c})$ are distinct members of $\mathcal{K}$. The dihedral group $\mathbb{D}_{n}=\mathbb{Z}_{n} \rtimes \mathbb{Z}_{2}$ acts on $D$, and hence on $\mathcal{K}$. Given $c \in \mathcal{K}$, we let $\mathbb{Z}_{n}(c) \subset \mathcal{K}$ denote its $\mathbb{Z}_{n}$-orbit.

The group of $\mathfrak{g}$-automorphisms has a semi-direct product

$$
\operatorname{aut}(\mathfrak{g})=\operatorname{int}(\mathfrak{g}) \rtimes \operatorname{aut}\left(D_{0}\right)
$$

where $\operatorname{int}(\mathfrak{g})$ consists of the inner automorphisms and is a normal subgroup, and aut $\left(D_{0}\right)$ is the automorphism group of the Dynkin diagram $D_{0}$ of $\mathfrak{g}$. We let $D_{0}$ denote the Dynkin diagram, because the notation $D$ has been used for the extended Dynkin diagram. See for instance [5, Thm.7.8]. Fix a Cartan subalgebra $\mathfrak{h}$ of $\mathfrak{g}$, and let $\Delta \subset \mathfrak{h}^{*}$ be its root system. We have the root space decomposition $\mathfrak{g}=\mathfrak{h}+\sum_{\Delta} \mathfrak{g}_{\alpha}$. Each member $\sigma$ of aut $(\mathfrak{g})$ which stabilizes $\mathfrak{h}$ induces an automorphism $\theta$ on $\Delta$, given by $\sigma \mathfrak{g}_{\alpha}=\mathfrak{g}_{\theta \alpha}$. So by considering $\mathfrak{g}$-automorphisms which stabilize $\mathfrak{h}$, (1) leads to

$$
\operatorname{aut}(\Delta)=W \rtimes \operatorname{aut}\left(D_{0}\right)
$$

where $W$ is the Weyl group of $\Delta$.

Given $\alpha \in \Delta$, it defines a reflection $R_{\alpha}: \Delta \longrightarrow \Delta$, where $R_{\alpha}$ maps $\alpha$ to $-\alpha$, and $R_{\alpha}(\beta)=\beta$ if $\beta$ is perpendicular to $\alpha$. Then $W$ is generated by the reflections $\left\{R_{\alpha} ; \alpha \in \Delta\right\}$ [3, III-9].

Let $c \in \mathcal{K}$, and let $m=\sum_{D} c_{j}$. As discussed in Theorem 1.1. it represents some $\mathfrak{g}$-automorphisms $\sigma$ of order $m$. This means that there exists $\Pi \subset \Delta$, which is a simple system together with its lowest root, such that the vertices of $D$ represent $\Pi$, and $\sigma$ has eigenvalue $\omega^{c_{j}}$ on the root space of $c_{j}$. Here $\omega=\exp (2 \pi i) / m$.

Suppose $c, d \in \mathcal{K}$ both represent $\sigma$, but with respect to $\Pi, \Pi^{\prime}$. Then we have $\Pi^{\prime}=g \Pi$ for some $g \in \operatorname{aut}(\Delta)$, because aut $(\Delta)$ acts transitively on the family of simple systems. If $g$ is induced by an inner automorphism, then $g \in W$. And since the reflections generate $W, g$ is the composite of a sequence of reflections $R_{1}, \ldots, R_{n}$. In this case we write $c \sim d$. We shall investigate the necessary and sufficient conditions for $c \sim d$.

Proposition 2.1. If $\mathbb{Z}_{n}(c)=\mathbb{Z}_{n}(d)$, then $c \sim d$.

Proof: Let $\alpha_{1}, \ldots, \alpha_{n}$ be the roots which are represented by the vertices of $D$. Let $R_{j}$ be the root reflection which maps $\alpha_{j}$ to $-\alpha_{j}$ and fixes the roots perpendicular to $\alpha_{j}$. We claim that

$$
\begin{array}{ll}
\text { (a) } & R_{2} \cdot \ldots \cdot R_{n}\left(\alpha_{j}\right)=\alpha_{j+1} \text { for } j=1, \ldots, n-1 \text {; } \\
\text { (b) } R_{2} \cdot \ldots \cdot R_{n}\left(\alpha_{n}\right)=\alpha_{1} \text {. }
\end{array}
$$


For $j=2, \ldots, n-1$,

$$
\begin{aligned}
R_{2} \cdot \ldots \cdot R_{n}\left(\alpha_{j}\right) & =R_{2} \cdot \ldots \cdot R_{j+1}\left(\alpha_{j}\right) \\
& =R_{2} \cdot \ldots \cdot R_{j}\left(\alpha_{j}+\alpha_{j+1}\right) \\
& =R_{2} \cdot \ldots \cdot R_{j-1}\left(\left(-\alpha_{j}\right)+\left(\alpha_{j+1}+\alpha_{j}\right)\right) \\
& =R_{2} \cdot \ldots \cdot R_{j-1}\left(\alpha_{j+1}\right)=\alpha_{j+1} .
\end{aligned}
$$

Also,

$$
\begin{aligned}
R_{2} \cdot \ldots \cdot R_{n}\left(\alpha_{1}\right) & =R_{2} \cdot \ldots \cdot R_{n-1}\left(\alpha_{1}+\alpha_{n}\right) \\
& =R_{2} \cdot \ldots \cdot R_{n-2}\left(\alpha_{1}+\alpha_{n-1}+\alpha_{n}\right) \\
& =R_{2} \cdot \ldots \cdot R_{n-3}\left(\alpha_{1}+\left(\alpha_{n-2}+\alpha_{n-1}\right)+\alpha_{n}\right) \\
& =\ldots=R_{2}\left(\alpha_{1}+\alpha_{3}+\ldots \alpha_{n}\right) \\
& =\left(\alpha_{2}+\alpha_{1}\right)+\left(\alpha_{2}+\alpha_{3}\right)+\alpha_{4}+\ldots+\alpha_{n} .
\end{aligned}
$$

Since $\sum_{1}^{n} \alpha_{j}=0$, the last line of (4) becomes $\alpha_{2}$. Thus (3) and (4) lead to (2) (a). We can also prove (2) (b) directly, or simply note that 2 (a) implies 2 (b). This proves (2) as claimed.

Let $\theta \in \mathbb{Z}_{n}$ be the 1-step counter-clockwise rotation on $D$. Namely it rotates the vertices by

$$
\theta: 1 \rightarrow 2 \rightarrow \ldots \rightarrow n \rightarrow 1 .
$$

By (2), $\theta=R_{2} \cdot \ldots \cdot R_{n}$. Hence $\theta c \sim c$. Since $\theta$ generates $\mathbb{Z}_{n}$, the proposition follows.

We shall illustrate the arguments of Proposition 2.1 by an example later (see Figure 2). Fix a positive integer $m>1$. Let

$$
\omega=\exp (2 \pi i / m) .
$$

We shall work on $\mathfrak{g}$-automorphisms of order $m$, and they are represented by Kac diagrams $c$ such that $\sum_{1}^{n} c_{j}=m$. The diagram $c$ represents the automorphism which acts as multiplication by $\omega^{c_{j}}$ on the root space of vertex $j$. Define

$$
\phi: \mathcal{K} \longrightarrow \mathbb{C} ; \phi(c)=\sum_{j=1}^{n} \omega^{c_{n}+\ldots+c_{j}} .
$$

Thus $\phi(c)=\omega^{c_{n}+\ldots+c_{1}}+\ldots+\omega^{c_{n}}=1+\omega^{c_{n}+\ldots+c_{2}}+\ldots+\omega^{c_{n}}$. Let

$$
S(c)=\left\{\omega^{c_{1}+\ldots+c_{j}} \phi(c) ; j=1, \ldots, n\right\} \subset \mathbb{C} .
$$

The next proposition shows that $S(c)$ is an invariance of rotations and equivalence relation on $\mathcal{K}$.

\section{Proposition 2.2.}

(a) $S(c)=\phi\left(\mathbb{Z}_{n}(c)\right)$.

(b) If $d \in \mathbb{Z}_{n}(c)$, then $S(c)=S(d)$.

(c) If $c \sim d$, then $S(c)=S(d)$.

Proof: Let $\theta \in \mathbb{Z}_{n}$ be the rotation (5), and let $d=\theta c$. Then $d_{1}=c_{n}$ and $d_{r}=c_{r-1}$ for all $r>1$. Therefore,

$$
\begin{aligned}
\phi(\theta c) & =1+\omega^{c_{n-1}+\ldots+c_{1}}+\omega^{c_{n-1}+\ldots+c_{2}}+\ldots+\omega^{c_{n-1}} \\
& =\omega^{-c_{n}}\left(\omega^{c_{n}}+1+\omega^{c_{n}+\ldots+c_{2}}+\ldots+\omega^{c_{n}+c_{n-1}}\right) \\
& =\omega^{-c_{n}} \phi(c) .
\end{aligned}
$$

Then $\phi\left(\theta^{2} c\right)=\omega^{-(\theta c)_{n}} \phi(\theta c)=\omega^{-c_{n-1}-c_{n}} \phi(c)$. Continue this process and get

$$
\phi\left(\theta^{j} c\right)=\omega^{-c_{n-j+1}-\ldots-c_{n}} \phi(c)=\omega^{c_{1}+\ldots+c_{n-j}} \phi(c) .
$$

Hence $S(c)=\left\{\omega^{c_{1}+\ldots+c_{j}}\right\}_{j} \cdot \phi(c)=\left\{\phi\left(\theta^{j} c\right)\right\}_{n-j}=\phi\left(\mathbb{Z}_{n}(c)\right)$, and part (a) follows.

By (6),

$$
S(d)=\left\{\omega^{d_{1}+\ldots+d_{r}}\right\}_{r=1}^{n} \cdot \phi(d)=\left(\left\{\omega^{c_{n}}\right\} \cup\left\{\omega^{c_{n}+c_{1}+\ldots+c_{r}}\right\}_{r=1}^{n-1}\right) \cdot \omega^{-c_{n}} \phi(c)=S(c) .
$$

Since $\theta$ generates $\mathbb{Z}_{n}$, this proves part (b) of the proposition. 
Next we prove part (c). If $c$ is a Kac diagram with respect to $\Pi \subset \Delta$ (where $\Pi$ is a simple system and its lowest root), we let $F_{j} c$ be the resulting Kac diagram with respect to $R_{j} \Pi$. We claim that

$$
S\left(F_{j} c\right)=S(c) ; j=1, \ldots, n .
$$

Let $j=2, \ldots, n-1$ and let $d=F_{j} c$. Then

$$
\begin{aligned}
& d_{r}=c_{r} \text { for all } r=1, \ldots, j-2, j+2, \ldots, n ; \\
& d_{j \pm 1}=c_{j \pm 1}+c_{j}, d_{j}=-c_{j} .
\end{aligned}
$$

By (8), $d_{j-1}+d_{j}+d_{j+1}=c_{j-1}+c_{j}+c_{j+1}$. Hence

$$
d_{r}+\ldots+d_{n}=c_{r}+\ldots+c_{n} \text { for all } r \leq j-1 \text { or } r>j+1 .
$$

Also,

$$
\omega^{d_{j}+d_{j \pm 1}}+\omega^{d_{j \pm 1}}=\omega^{-c_{j}+\left(c_{j \pm 1}+c_{j}\right)}+\omega^{c_{j \pm 1}+c_{j}}=\omega^{c_{j \pm 1}}+\omega^{c_{j \pm 1}+c_{j}} .
$$

By 9 and 10, we have $\left\{\omega^{d_{1}+\ldots d_{r}}\right\}_{r=1}^{n}=\left\{\omega^{c_{1}+\ldots c_{r}}\right\}_{r=1}^{n}$ as well as

$$
\phi(d)=\sum_{1}^{n} \omega^{d_{r}+\ldots+d_{n}}=\sum_{1}^{n} \omega^{c_{r}+\ldots+c_{n}}=\phi(c) .
$$

This implies that

$$
S(d)=\left\{\omega^{d_{1}+\ldots+d_{r}}\right\}_{r=1}^{n} \cdot \phi(d)=\left\{\omega^{c_{1}+\ldots+c_{r}}\right\}_{r=1}^{n} \cdot \phi(c)=S(c) .
$$

This proves (7) for $j=2, \ldots, n-1$.

One can also check directly (7) for $j=1$ and $j=n$. Alternatively, for $j=1, n$, there exists a rotation $g$ and $k \in\{2, \ldots, n-1\}$ such that $F_{j} c=g^{-1} F_{k} g c$. Then by part (b) of this proposition, $S\left(F_{j} c\right)=S\left(g^{-1} F_{k} g c\right)=$ $S(c)$. This completes the proof of $(7)$, which leads to part (c) of this proposition.

An element of $\mathbb{D}_{n} \backslash \mathbb{Z}_{n}$ of order 2 is called a reflection. We say that a Kac diagram $c$ is symmetric if there exists a reflection $r$ such that $r c=c$. Equivalently for any $z \in \mathbb{D}_{n} \backslash \mathbb{Z}_{n}$, there exists $g \in \mathbb{Z}_{n}$ such that $z c=g c$. Let $\overline{S(c)}$ denote the complex conjugation on the elements of $S(c) \subset \mathbb{C}$.

Proposition 2.3. If $S(c)=\overline{S(c)}$, then $c$ is symmetric.

Proof: Use the polar coordinates to write $r e^{i t} \in \mathbb{C}^{\times}$, where $r>0$ and $t \in[0,2 \pi)$. Define a partial order on $\mathbb{C}^{\times}$by $r e^{i t} \succeq r^{\prime} e^{i t^{\prime}}$ if $t \geq t^{\prime}$.

Suppose that $S(c)=\overline{S(c)}$. By Proposition $2.2(\mathrm{~b})$, if $c^{\prime} \in \mathbb{Z}_{n}(c)$, then $S\left(c^{\prime}\right)=S(c)$. Furthermore $c$ is symmetric if and only if $c^{\prime}$ is symmetric. Therefore, replacing $c$ by another member in $\mathbb{Z}_{n}(c)$ if necessary, we may assume that $\phi(c) \preceq \phi\left(c^{\prime}\right)$ for all $c^{\prime} \in \mathbb{Z}_{n}(c)$. The elements of $S(c)$ are

$$
\phi(c) \preceq \omega^{c_{1}} \phi(c) \preceq \omega^{c_{1}+c_{2}} \phi(c) \preceq \ldots \preceq \omega^{c_{1}+\ldots+c_{n-1}} \phi(c) .
$$

Case 1: $\phi(c) \in \mathbb{R}$.

Since $\omega^{c_{1}+\ldots+c_{n}}=1$, by 11 , the elements of $\overline{S(c)}$ are

$$
\phi(c) \preceq \omega^{c_{n}} \phi(c) \preceq \omega^{c_{n}+c_{n-1}} \phi(c) \preceq \ldots \preceq \omega^{c_{n}+\ldots+c_{2}} \phi(c) .
$$

Since $S(c)=\overline{S(c)}$, by 11 and 12 , we have $c_{1}=c_{n}, c_{2}=c_{n-1}$ and more generally $c_{j}=c_{n-j+1}$. Then $c$ is symmetric by the reflection $r(j)=n-j+1$. Case 2: $\phi(c) \notin \mathbb{R}$.

Since $\omega^{c_{1}+\ldots+c_{n}}=1$, by 11 , the elements of $\overline{S(c)}$ are

$$
\omega^{c_{n}} \overline{\phi(c)} \preceq \omega^{c_{n}+c_{n-1}} \overline{\phi(c)} \preceq \omega^{c_{n}+\ldots+c_{2}} \overline{\phi(c)} \preceq \overline{\phi(c)} .
$$

Since $S(c)=\overline{S(c)}$, by 11 and $\sqrt{13}$, we have $\phi(c)=\omega^{c_{n}} \overline{\phi(c)}, \omega^{c_{1}} \phi(c)=\omega^{c_{n}+c_{n-1}} \overline{\phi(c)}$ and more generally

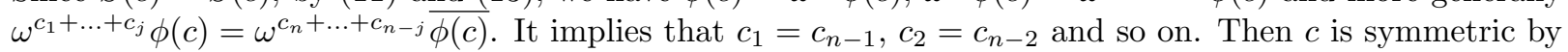
the reflection $r(j)=n-j$. 
Proposition 2.4. For any $z \in \mathbb{D}_{n} \backslash \mathbb{Z}_{n}$, we have $S(z c)=\overline{S(c)}$.

Proof: A specific reflection $r$ is given by $1 \leftrightarrow n, 2 \leftrightarrow n-1$, and more generally

$$
r(j)=n-j+1 .
$$

For such $r$ and any $c \in \mathcal{K}$,

$$
\begin{aligned}
\phi(r c) & =\omega^{c_{1}+\ldots+c_{n}}+\omega^{c_{1}+\ldots+c_{n-1}}+\ldots+\omega^{c_{1}} \\
& =1+\omega^{-c_{n}}+\omega^{-c_{n}-c_{n-1}}+\ldots+\omega^{-c_{n}-\ldots-c_{2}} \\
& =1+\overline{\omega^{c_{n}}}+\overline{\omega^{c_{n}+c_{n-1}}}+\ldots+\overline{\omega^{c_{n}+\ldots+c_{2}}} \\
& =\overline{\phi(c)} .
\end{aligned}
$$

Recall that $S(c)=\left\{\omega^{c_{1}+\ldots+c_{j}} \phi(c)\right\}_{j=1}^{n}$. We have

$$
\begin{array}{rlrl}
\overline{S(c)} & =\left\{\omega^{c_{j+1}+\ldots+c_{n}} \overline{\phi(c)}\right\}_{j=1}^{n} & \text { as } \omega^{c_{1}+\ldots+c_{n}}=1 \\
& =\left\{\omega^{(r c)_{1}+\ldots+(r c)_{j}} \phi(r c)\right\}_{j=1}^{n} & \text { by } 14 \text { and } 15 \\
& =S(r c) .
\end{array}
$$

An arbitrary element of $\mathbb{D}_{n} \backslash \mathbb{Z}_{n}$ is of the form $r g$, where $g \in \mathbb{Z}_{n}$. By Proposition 2.2(b) and (16), $S(r g c)=$ $\overline{S(g c)}=\overline{S(c)}$.

\section{Proof of Theorem 1.2:}

Let $c, d \in \mathcal{K}$ be related by the diagram symmetry $\mathbb{D}_{n}$. By Proposition 2.1 if there exists a rotation $g \in \mathbb{Z}_{n}$ such that $g c=d$, then $c \sim d$.

Conversely, suppose that $r c=d$ for some $r \in \mathbb{D}_{n} \backslash_{\mathbb{Z}}$, but $c$ and $d$ are not related by the rotation group $\mathbb{Z}_{n}$. Then $c$ is not symmetric, so by Proposition 2.3. $S(c) \neq \overline{S(c)}$. By Proposition 2.4 $S(c) \neq S(d)$. By Proposition 2.2, $c \nsim d$. This proves Theorem 1.2 .

\section{Examples}

We provide an example to illustrate the concepts discussed earlier. Let $\mathfrak{g}=\mathfrak{s l}(3, \mathbb{C})$. Its extended Dynkin diagram $D$ is given by Figure 2(a), namely a triangle. We denote its vertices by $x, y, z$, and they represent a simple system with lowest root as indicated in Figure 2(a).

Consider the Kac diagram $c$ in Figure 2(b), where $c_{x}=1, c_{y}=2$ and $c_{z}=0$. It represents a $\mathfrak{g}$-automorphism $\sigma$ of order $c_{x}+c_{y}+c_{z}=3$. Let $\omega=\exp (2 \pi i / 3)$. Then all the root spaces are eigenspaces of $\sigma$, with eigenvalues $1, \omega$ or $\omega^{2}$. We indicate these eigenvalues with the roots in Figure 2(b).

Consider the reflection $R_{x}$, which maps $x$ to $-x$ and fixes the hyperplane $x^{\perp}$. The hyperplane is just the dotted line in Figure 2(b), so $R_{x}$ is the reflection about the dotted line. Figure 2(c) show the resulting eigenvalues after performing $R_{x}$. The corresponding Kac diagram is given in Figure 2(c). By comparing the Kac diagrams of Figure 2(b) and Figure 2(c), we see that they are related by a rotation on the triangle. This observation verifies Proposition 2.1, namely the Kac diagrams in Figure 2(b) and Figure 2(c) are related by rotation (i.e. they are in the same $\mathbb{Z}_{3}$-orbit), and indeed they are equivalent because they are related by performing $R_{x}$ to the root system.

Next we classify all inner automorphisms on $\mathfrak{g}=\mathfrak{s l}(3, \mathbb{C})$ of order 3 , up to conjugation by inner automorphisms. All Kac diagrams are assignments of nonnegative integers $\left\{c_{1}, c_{2}, c_{3}\right\}$ without nontrivial common factor such that $c_{1}+c_{2}+c_{3}=3$. So the only possibilities are $\{1,1,1\}$ and $\{0,1,2\}$.

Figure 3 provides all possibilities, where two diagrams are not distinguished if they are related by a rotation $\mathbb{Z}_{3}$. So there are three such $\mathfrak{g}$-automorphisms. Note that Figure 3(b) and Figure 3(c) are related by a diagram reflection but not rotation, so they represent order-3 automorphisms $\sigma, \tau \in$ aut $(\mathfrak{g})$ such that $\sigma=u^{-1} \tau u$ for some $u \in \operatorname{aut}(\mathfrak{g})$, but $u \notin \operatorname{int}(\mathfrak{g})$. So $\sigma, \tau$ are not distinguished by Kac's Theorem 1.1, but are distinguished by our stricter Theorem 1.2 Diagram rotations are not needed, as discussed in Figure 2 above. So Figure 3 exhausts all possibilities up to conjugation by inner automorphisms. 

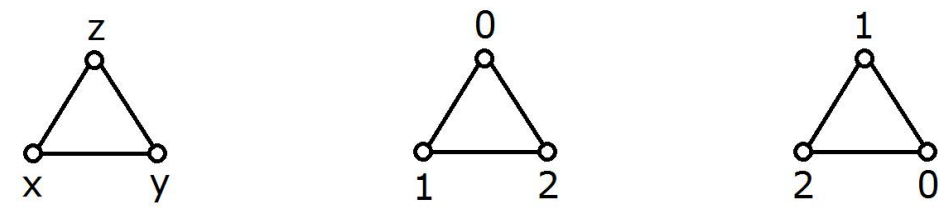

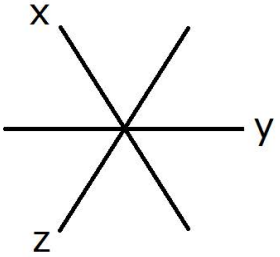

(a)

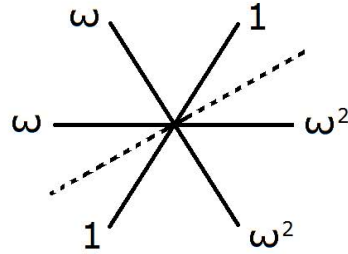

(b)

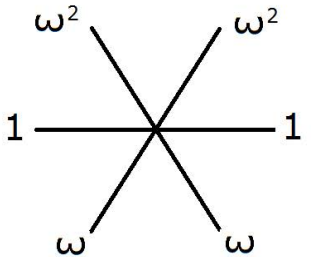

(c)

Figure 2: Reflection and Kac diagrams.

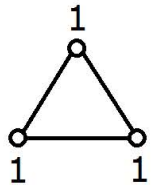

(a)

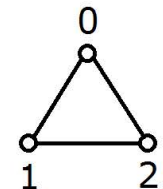

(b)

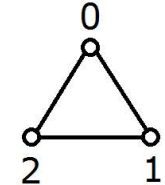

(c)

Figure 3: Automorphisms of order 3 on $\mathfrak{s l}(3, \mathbb{C})$.

\section{References}

[1] Chuah, M. K., 2012, Finite order automorphisms on contragredient Lie superalgebras, Journal of Algebra, 351, 138-159.

[2] Helgason, S., 2001, Differential Geometry, Lie Groups, and Symmetric Spaces, Graduate Studies in Mathematics 34, American Mathematical Society, Providence.

[3] Humphreys, J. E., 1972, Introduction to Lie Algebras and Representation Theory, Springer-Verlag, New York.

[4] Kac, V. G., 1990, Infinite Dimensional Lie Algebras, 3rd. ed., Cambridge University Press, Cambridge.

[5] Knapp, A. W., 2002, Lie groups beyond an introduction, 2nd. ed., Progress in Mathematics 140, Birkhäuser, Boston. 